\title{
Stability and Control Derivative Estimation for the Bell-Shaped Lift Distribution
}

\author{
Loren J. Newton \\ The University of California, Berkeley \\ NASA Armstrong Flight Research Center
}




\section{Introduction: The Bell-Shaped Lift Distribution}

- Ludwig Prandtl, 1933

- Minimum induced drag solution for a wing of constrained mass

- Results:

- $11 \%$ less induced drag, $22 \%$ greater span than the elliptical spanload (solution for a wing of defined span)

- Upwash at the wingtips

- Proverse yaw \& tailless flight

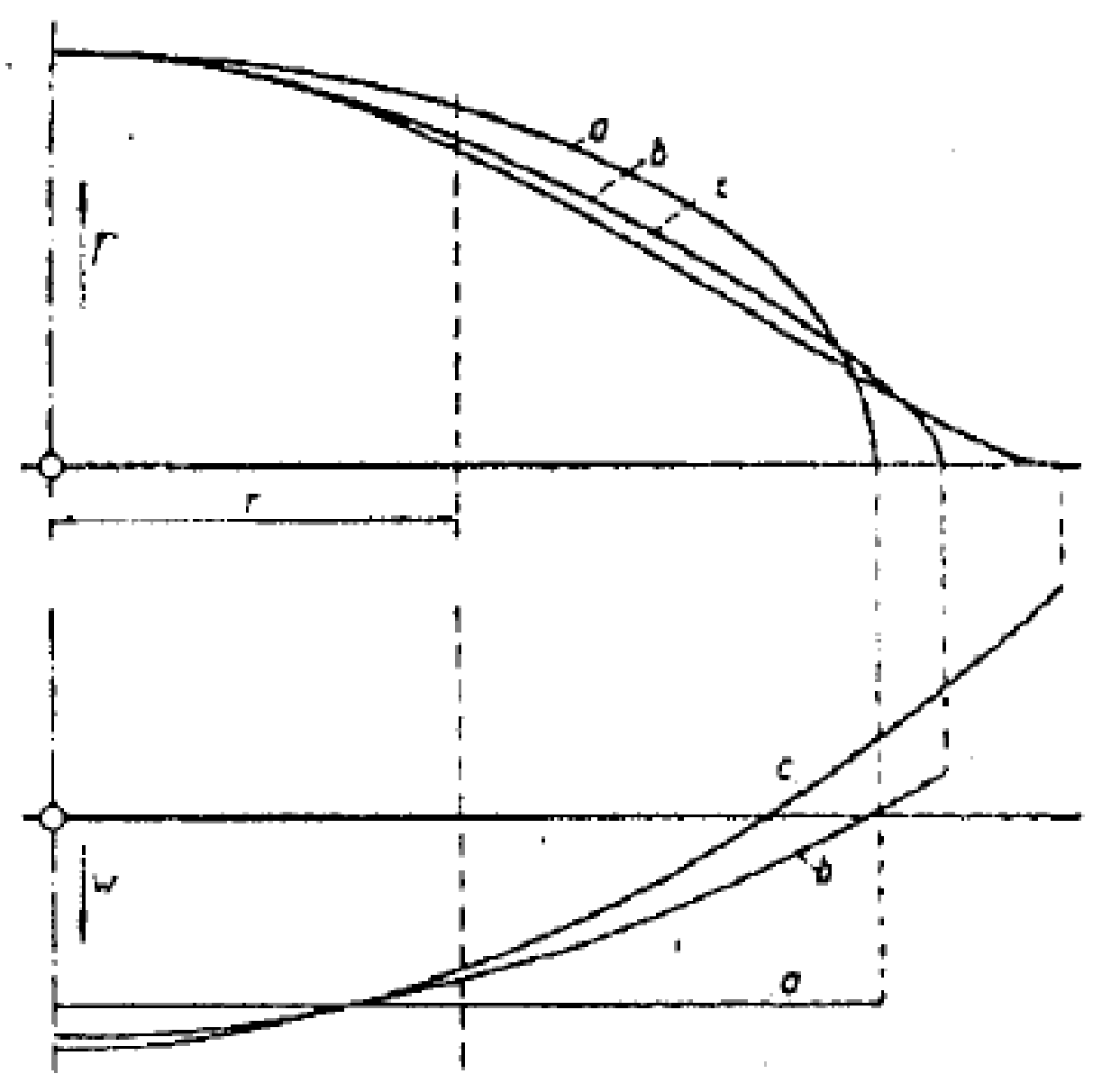




\section{Introduction: PRANDTL-D}

- Preliminary Research AerodyNamic Design To Lower Drag

- Uninhabited, unpowered flying wings with the Bell-Shaped Lift Distribution

- Prandtl-1: Lightly instrumented proof of concept (12.3' span)

- Prandtl-2: Flight computer-equipped data acquisition (12.3' span)

- Prandtl-3: Pressure/strain data for spanload measurement (25' span) 


\section{Flight Test Procedures}

- Edwards AFB lakebeds

- Average flight $~ 90 \mathrm{sec}$.

- Elastic high-start launch

- Doublet maneuvers: square wave input to control surfaces

- Pitch

- Roll

-2-3 doublets per flight

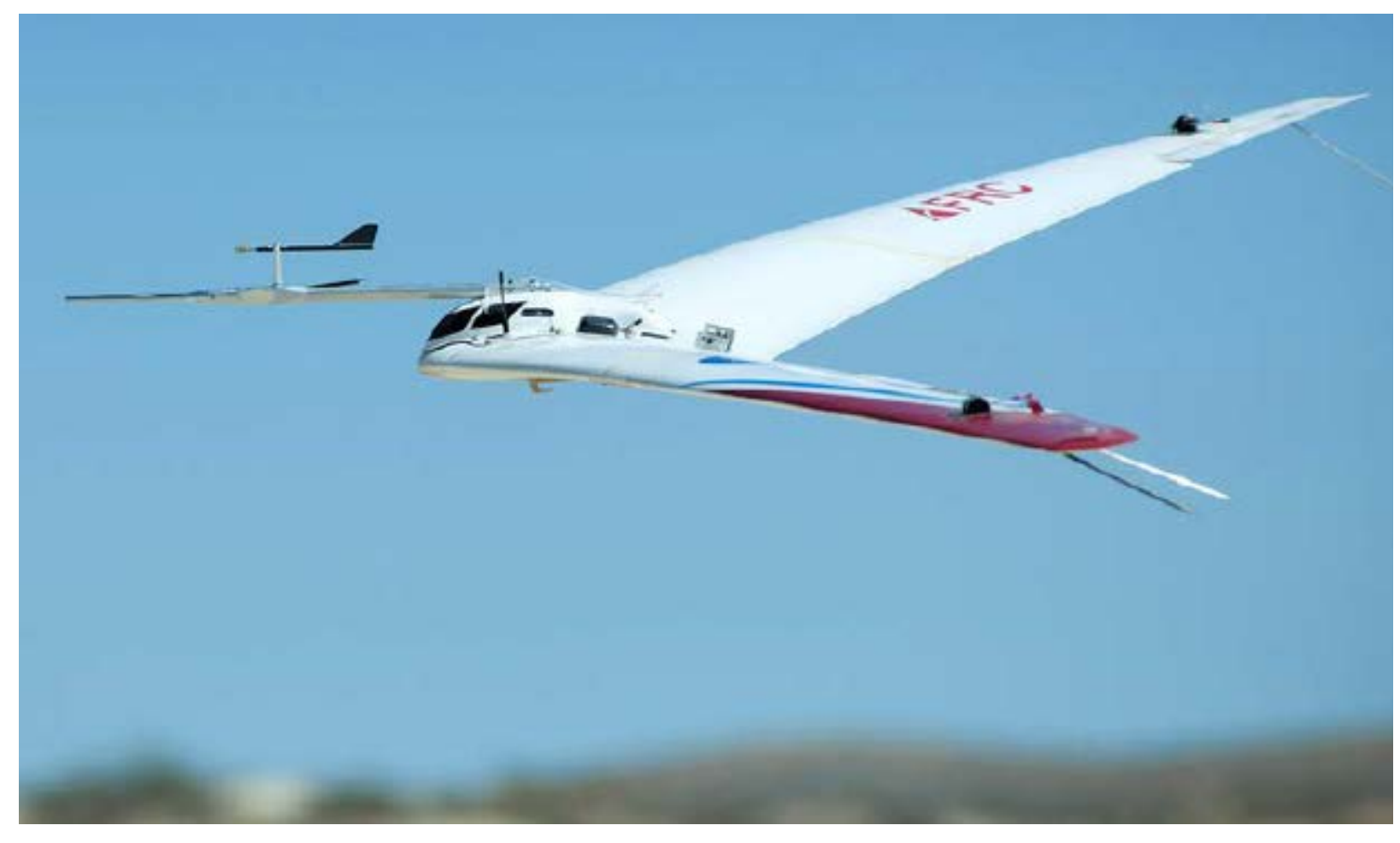




\section{Flight Dynamics}

$$
\begin{gathered}
\dot{V}=-\frac{\bar{q} s}{m} C_{D}+g(\cos \phi \cos \theta \sin \alpha \cos \beta+\sin \phi \cos \theta \sin \beta-\sin \theta \cos \alpha \cos \beta) \\
\dot{\alpha}=q-\tan \beta(p \cos \alpha+r \sin \alpha)-\frac{\bar{q} s R}{m V \cos \beta} C_{L}+\frac{g R}{V \cos \beta}(\cos \theta \cos \phi \cos \alpha+\sin \theta \sin \alpha) \\
\dot{\beta}=p \sin \alpha-r \cos \alpha+\frac{\bar{q} s R}{m V} C_{Y}+\frac{g R}{V}[\cos \beta \cos \theta \sin \phi-\sin \beta(\cos \theta \cos \phi \sin \alpha-\sin \theta \cos \alpha)] \\
I_{x} x \dot{p}-I_{x y} \dot{q}-I_{x z} \dot{r}=\bar{q} s b C_{l} R+\left[q r\left(I_{y} y-I_{z} z\right)+\left(q^{2}-r^{2}\right) I_{y z}+p q I_{x z}-p r I_{x y}\right] / R \\
I_{y} y \dot{q}-I_{y z} \dot{r}-I_{x y} \dot{p}=\bar{q} s b C_{m} R+\left[p r\left(I_{z} z-I_{x} x\right)+\left(r^{2}-p^{2}\right) I_{x z}+q r I_{x y}-p q I_{y z}\right] / R \\
I_{z} z \dot{r}-I_{x z} \dot{p}-I_{y z} \dot{q}=\bar{q} s b C_{n} R+\left[p q\left(I_{x} x-I_{y} y\right)+\left(p^{2}-q^{2}\right) I_{x y}+p r I_{y z}-q r I_{x z}\right] / R \\
\dot{\theta}=q \cos \phi-r \sin \phi \\
\dot{\phi}=p+\tan \theta(r \cos \phi+q \sin \phi)
\end{gathered}
$$




\section{Flight Dynamics}

$$
\begin{gathered}
C_{A}=C_{A_{0}}+C_{A_{\alpha}} \alpha+\frac{c}{2 V R} C_{A_{q}} q+C_{A_{\delta e}} \delta e \\
C_{N}=C_{N_{0}}+C_{N_{\alpha}} \alpha+\frac{C}{2 V R} C_{N_{q}} q+C_{N_{\delta e}} \delta e \\
C_{m}=C_{m_{0}}+C_{m_{\alpha}} \alpha+\frac{c}{2 V R} C_{m_{q}} q+C_{m_{\delta e}} \delta e \\
C_{Y}=C_{Y_{0}}+C_{Y_{\beta}} \beta+\frac{b}{2 V R}\left(C_{Y_{p}} p+C_{Y_{r}} r\right)+C_{Y_{\delta a}} \delta a \\
C_{l}=C_{l_{0}}+C_{l_{\beta}} \beta+\frac{b}{2 V R}\left(C_{l_{p}} p+C_{l_{r}} r\right)+C_{l_{\delta a}} \delta a \\
C_{n}=C_{n_{0}}+C_{n_{\beta}} \beta+\frac{b}{2 V R}\left(C_{n_{p}} p+C_{n_{r}} r\right)+C_{n_{\delta a}} \delta a
\end{gathered}
$$




\section{Parameter Estimation}

- Method for determining stability and control derivatives from flight data

- Derivatives are varied in the aircraft equations of state until the mathematical model matches recorded flight data

- NASA Dryden code: MATLAB pEst MX.96
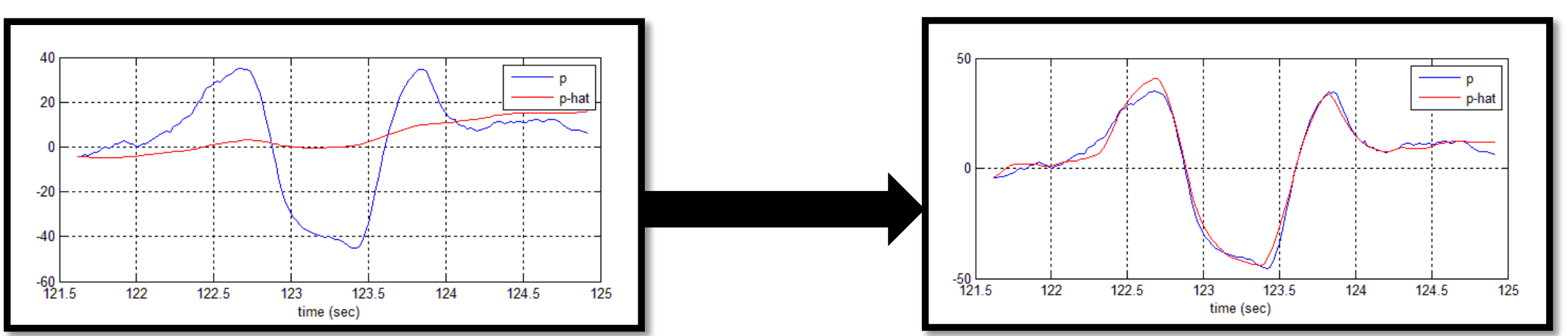


\section{Flight Data Conversion}

- Isolate doublets in data time histories

- Adjust units to pEst convention

- Correct axes and signs to flight control/pEst convention

- Define constants (geometry, mass properties) to $\mathrm{pEst}$

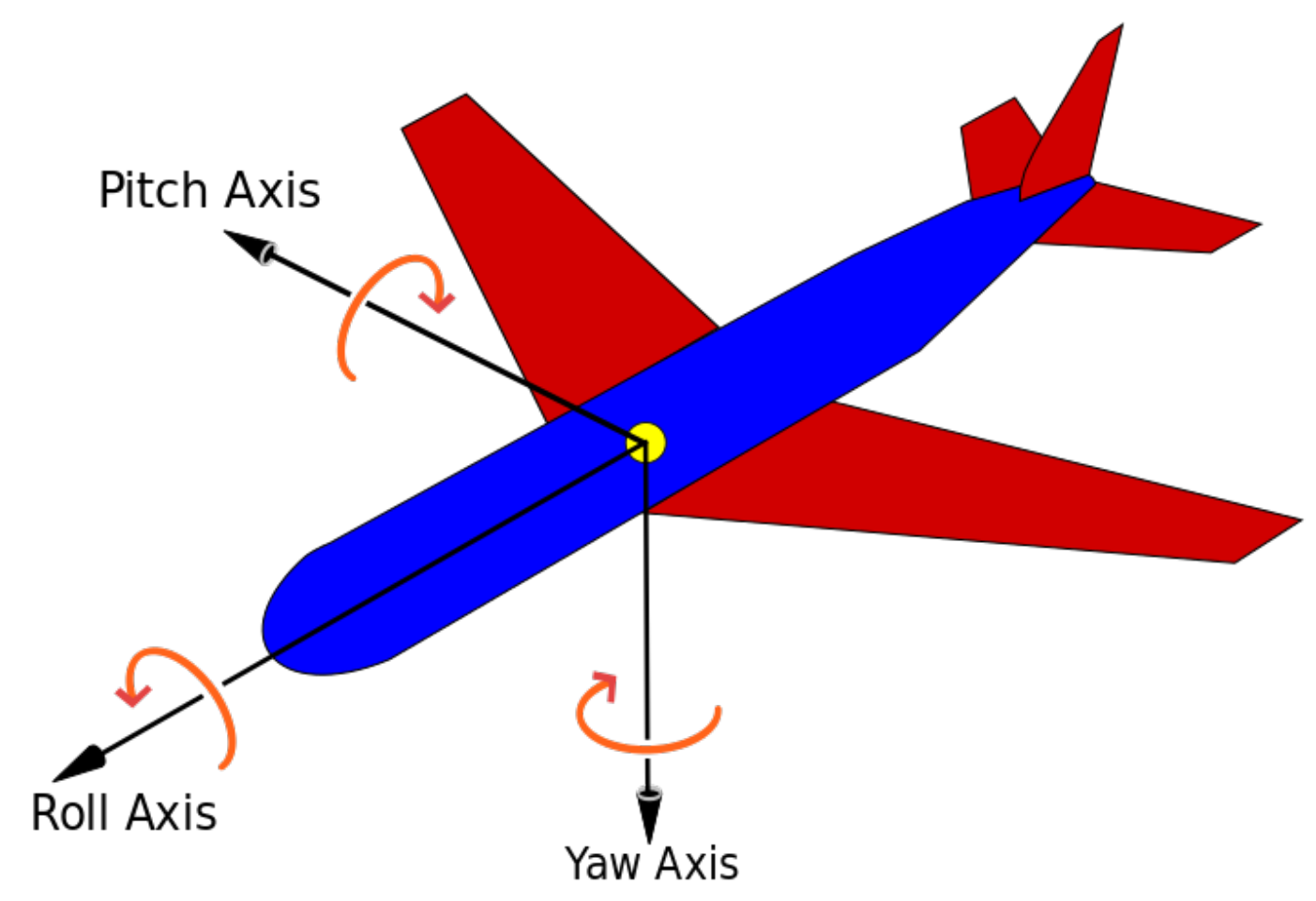




\section{Stability \& Control Derivative Estimation}

- Different pEst scripts for lateral and longitudinal maneuvers

- Lateral: estimated $\beta, p, r, a_{y}$ signals

- Longitudinal: estimated $\alpha, q, a_{n}$ signals

- User input: selecting estimating weights $W_{i i}$ for each signal $i$

- Algorithm minimizes cost function: summed squared difference between flight data and model estimate, scaled by $W$

$$
J=\frac{1}{2 n_{z} n_{t}} \sum_{i=1}^{n_{t}}\left[z\left(t_{i}\right)-\tilde{z}\left(t_{i}\right)\right]^{T} W\left[z\left(t_{i}\right)-\tilde{z}\left(t_{i}\right)\right]
$$




\section{Stability \& Control Derivative Maps}

- Prandtl-2 flew entirely in the linear regime

- Linear regressions were created for each S\&C derivative with respect to $\alpha$

- Data points were weighted by the inverse of the Cramer-Rao bound error estimated by pEst

- Applicable to lookup tables in simulation 


\section{Results: Unique Flight Dynamics}

- $C_{n \delta a}$ : nondimensional yawing moment due to aileron deflection

- Quantifies how the aircraft responds in yaw due to a roll command

- Sign specifies nature of yaw/roll coupling

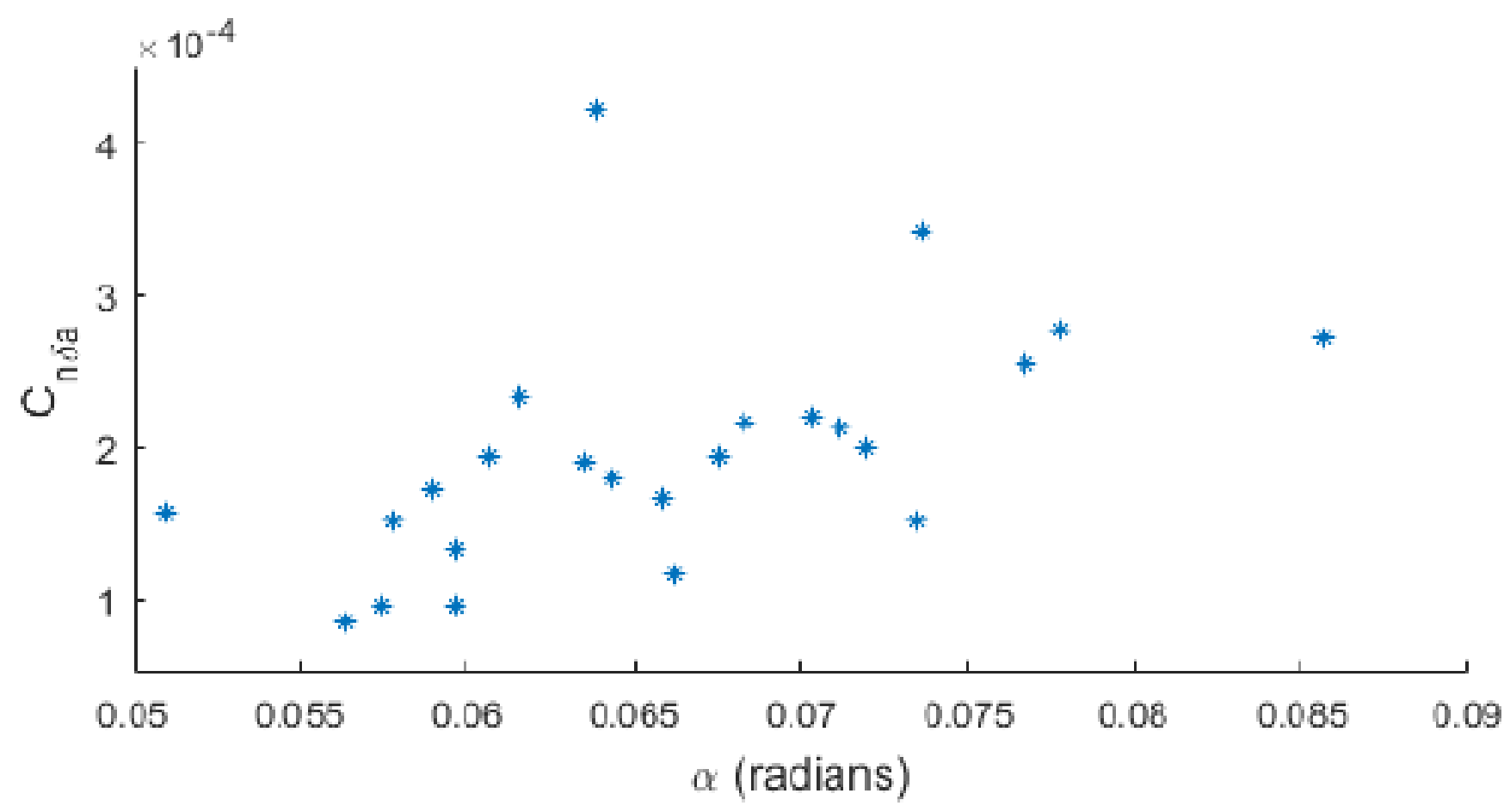




\section{Results: Algorithmic User Input}

- Demonstrated algorithmic weight selection to accelerate analysis

- Normalize square of signals by $W_{i i}=[\text { range }(i)]^{-2}$

- Cost function distributes error evenly as a percent error of each signal

- 2 data analysis teams: 1 algorithmic weight selection, 1 iterative "trial and error" weight selection 


\section{Results: Algorithmic User Input}

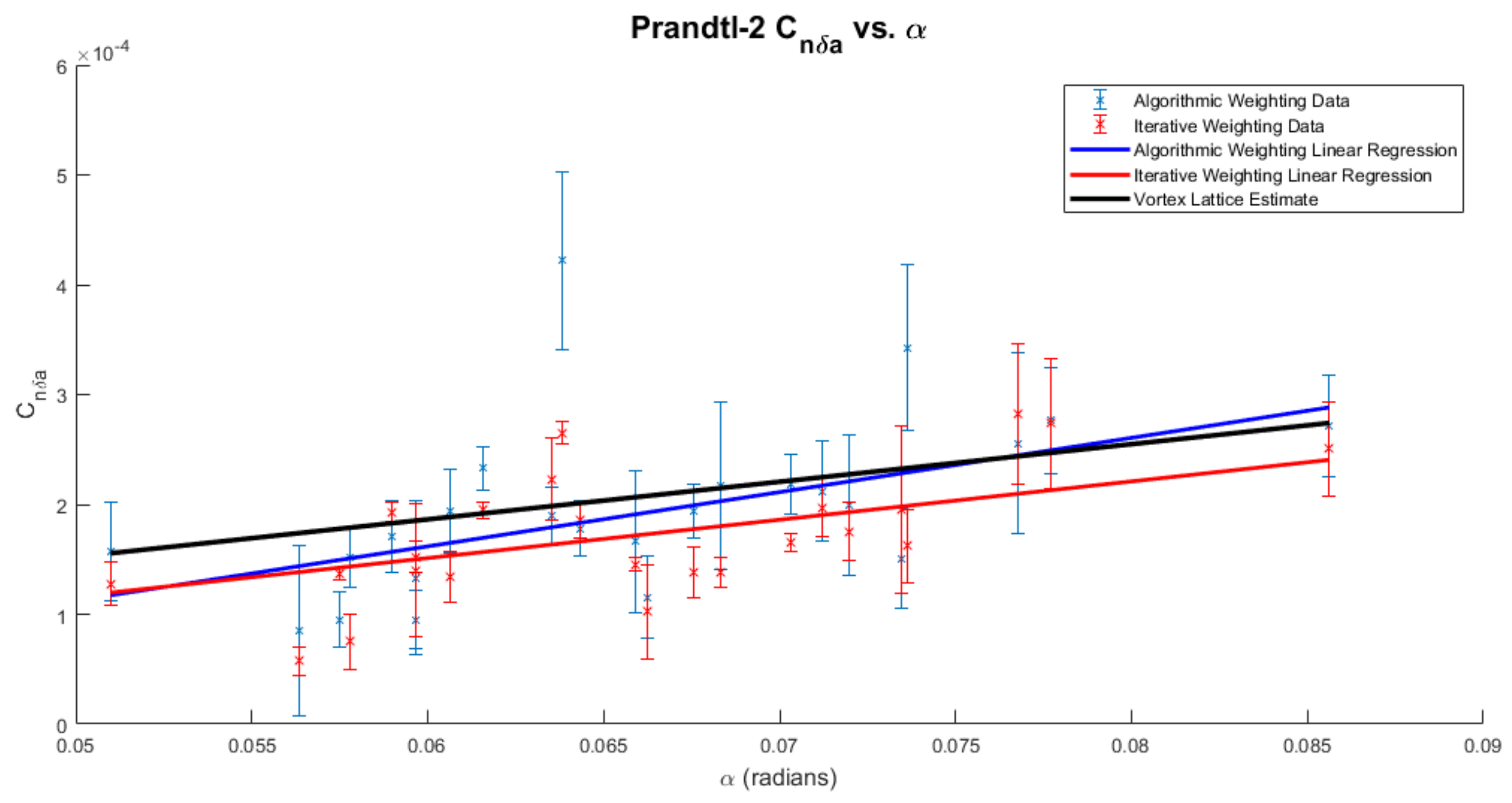




\section{Conclusions \& Future Steps}

- Prandtl-2 flight testing returned sufficient flight data to quantify the flight dynamics of the Bell-Shaped Lift Distribution equipped vehicle.

- Parameter Estimation was used to determine, from flight data, the characteristic stability and control derivatives. Two different teams with different weight selection schemes produced agreeing results.

- A positive $C_{n \delta a}$ provided quantifiable evidence of proverse yaw.

- Potential future steps: Prandtl-3 spanload measurements, PRANDTL-D flight dynamics simulator, autopilot development 


\section{Acknowledgements}

- Albion Bowers and Oscar Murillo, internship mentors at NASA Armstrong.

- Keenan Albee, for help with developing and running the analysis script.

Thank you! 


\section{Questions?}




\section{References}

- Bowers, A. H., and Murillo, O. J., "On Wings of the Minimum Induced Drag: Spanload Implications for Aircraft and Birds," NASA TP-2016-219072, 2016.

- Etkin, B., and Reid, L.D., Dynamics of Flight: Stability and Control, 3rd ed., Wiley \& Sons, Inc., Toronto, 1996, Chap. 5.

- Maine, R. E., and Iliff, K. W., "Application of Parameter Estimation to Aircraft Stability and Control," NASA RP-1168, 1986.

- Murray, J. E., and Maine, R. E., "pEst Version 2.1 User's Manual," NASA TM-82280, 1987.

- Prandtl, L., "Aplications of Modern Hydrodynamics to Aeronautics," NACA Report 116, 1921.

- Prandtl, L., "Regarding Wings with Minimum Induced Drag," 1933. 


\section{Appendix: Nomenclature}

- $A=$ axial force

- $b=$ reference span

- $C_{i}=$ nondimensional coefficient of $\bullet \quad n=$ yawing moment force or moment $i$

- $C_{m n}=$ nondimensional stability/control derivative: coefficient of $m$ due to $n$

- $c=$ reference chord

- $D=$ drag force

- $g$ = gravitational acceleration

- $I_{j k}=$ moment of inertia

- $L=$ lift force

- $I=$ rolling moment

- $M=$ vehicle mass
- $m=$ pitching moment

- $N=$ normal force

- $n_{t}=$ number of time steps

- $n_{z}=$ number of signals

- $o=$ coefficient bias

- $p=$ roll rate

- $q=$ pitch rate

- $\bar{q}=$ dynamic pressure

- $R=$ conversion parameter: $180 / \pi$

- $r$ = yaw rate

- $s=$ reference area

- $V=$ equivalent airspeed
- $W=$ weighting matrix

- $Y=$ side force

- $z=$ measured signal

- $\tilde{z}=$ estimated signal

- $\alpha=$ angle of attack

- $\beta=$ angle of sideslip

- $\xi=$ set of signal/estimate pairs

- $\varphi=$ roll angle

- $\theta=$ pitch angle

- $\psi=$ yaw angle

- $\delta e=$ elevator deflection

- $\delta a=$ aileron deflection 